\title{
Exosome-derived miRNAs as predictive biomarkers for diffuse large B-cell lymphoma chemotherapy resistance
}

\author{
Yuhua Feng ${ }^{1}$, Meizuo Zhong ${ }^{1}$, Shan Zeng ${ }^{1}$, Leyuan Wang ${ }^{2}$, Ping Liu ${ }^{1}$, Xiangyu Xiao ${ }^{1}$ \& \\ Yiping Liu*,1 \\ ${ }^{1}$ Department of Oncology, Xiangya Hospital, Central South University, Changsha 410008, Hunan, PR China \\ ${ }^{2}$ Department of Pediatrics, Xiangya Hospital, Central South University, Changsha 410008, Hunan, PR China \\ *Author for correspondence: Tel.: +86 1511633 1808; liuyiping@csu.edu.cn
}

\begin{abstract}
Aim: To analyze the expression profiles, clinicopathological features and chemotherapeutic efficacies of exosome-derived miRNAs in diffuse large B-cell lymphoma (DLBCL). Materials \& methods: Next-generation sequencing technique was performed to identify miRNA profiles in exosomes from parental and chemoresistant DLBCL cells. The results were validated by quantitative real-time PCR, and further analyzed by bioinformatics and statistical methods. Results: We identified 37 significantly upregulated and 17 downregulated miRNAs. Of four upregulated miRNAs validated, we found miR-99a-5p and miR-125b-5p were significantly upregulated. Increased levels of exosomal miR-99a-5p and miR-125b-5p in DLBCL patients' serum were associated with shorter progression-free survival time, and they can predict chemotherapeutic efficacy. Conclusion: Exosomal miR-99a-5p and miR-125b-5p can serve as biomarkers for DLBCL.
\end{abstract}

First draft submitted: 28 July 2018; Accepted for publication: 28 August 2018; Published online: 13 September 2018

Keywords: chemotherapy resistance $\bullet$ diffuse large B-cell lymphoma $\bullet$ exosomes $\bullet$ miRNA

Diffuse large B-cell lymphoma (DLBCL) is an aggressive malignant lymphoma that accounts for $35-40 \%$ of all non-Hodgkin lymphoma [1]. According to the 2008 WHO classification, DLBCL subtypes are classified according to gene expression profiles into the - activated B-cell-like subtype, germinal center B cell (GCB) like subtype, and not otherwise specified subtype [2]. Recently, researchers identified four genetic subtypes in DLBCL, termed MCD (based on the co-occurrence of the MYD88L265P and CD79B mutations), BN2 (based on BCL6 fusions and NOTCH2 mutations), N1 (based on NOTCH1 mutations) and EZB (based on EZH2 mutations and BCL2 translocations) [3]. The standard treatment for DLBCL patients is the R-CHOP regimen (cyclophosphamide, doxorubicin, vincristine and prednisone, combined with the anti-CD20 monoclonal antibody - rituximab). Although DLBCL can be cured in more than $50 \%$ of cases, up to a third of patients have relapse/refractory disease due to chemoresistance $[4,5]$. The existing prognostic measurement - the International Prognostic Index (IPI) can predict only survival time but not treatment efficacy [1]. Identifying new targets and treatment approaches to enhance DLBCL chemosensitivity is an urgent need.

miRNAs are small, single-stranded noncoding RNAs of 19-24 nt in length [6]. miRNAs function at the posttranscriptional level, and they are involved in many biological processes, such as cell proliferation and apoptosis [7]. Moreover, miRNAs have been found to be closely related to cancer chemosensitivity $[8,9]$.

Exosomes are small, lipid bilayer vesicles that are secreted by almost all types of cells [10]. Exosomes are approximately 40-100 nm in diameter, and contain proteins, miRNAs, mRNAs - among others [11]. Exosomes enhance cellular connections by transferring functional proteins, miRNAs and other substances [12]. Exosomes may play multiple roles in tumor development and therapy sensitivity. A study showed that in DLBCL, exosomes attenuated chemotherapeutic efficacy by encapsulating doxorubicin and removing it from the cells [13]. However, the role of exosomal miRNAs in DLBCL chemoresistance has not been elucidated.

In this study, we established an R-CHOP-resistant DLBCL cell line - referred to as SU-DHL-2/R and performed a next-generation sequencing (NGS) technique to identify differentially expressed miRNAs in exosomes from SU-

Future Medicine 
DHL-2/R cells and the parental SU-DHL-2 cell line. Then, we detected significant miRNAs by quantitative real-time PCR (qRT-PCR), analyzed their relationships with the clinicopathological features of DLBCL patients and evaluated their ability to predict treatment efficacy.

\section{Materials \& methods}

\section{Cell lines \& patient samples}

The human DLBCL cell line SU-DHL-2 (ATCC ${ }^{\circledR}$ CRL-2956 ${ }^{\mathrm{TM}}$ ) was obtained from the American Type Culture Collection (ATCC) (VA, USA). The cells were cultured in RPMI 1640 medium (HyClone, GE Healthcare Life Sciences, Logan, UT, USA) supplemented with 10\% fetal bovine serum (FBS; Gibco, CA, USA), 100 units $/ \mathrm{ml}$ penicillin and $100 \mathrm{mg} / \mathrm{ml}$ streptomycin (HyClone, GE Healthcare Life Sciences, UT, USA) in a humidified atmosphere of at $37^{\circ} \mathrm{C}$ with $5 \% \mathrm{CO}_{2}$.

Serum samples of DLBCL patients $(\mathrm{n}=116)$ from Xiangya Hospital, Central South University were collected between June 2015 and August 2016, and stored at $-80^{\circ} \mathrm{C}$ until used for exosomes isolation. Each patient's clinical information, including gender, age, IPI score, B symptoms, LDH level, Ann Arbor stage and treatment efficacy, was collected. All patients had complete clinical and follow-up data. According to the chemotherapy efficacy (evaluated by RECIST standard 1.1) after four cycles of R-CHOP (cyclophosphamide, doxorubicin, vincristine and prednisone, combined with rituximab) regimen, we divided patients into two groups - the chemosensitive (efficacy evaluation of complete response or partial response, $\mathrm{n}=83$ ) and chemoresistant (efficacy evaluation of stable disease or progressive disease, $\mathrm{n}=33$ ) groups. All samples were collected in accordance with the ethical standards of the responsible committees on human experimentation (institutional and national) and with the Helsinki Declaration of 1975, which was revised in 2008. All DLBCL patients were followed-up from the day of diagnosis to May 2018, and no patients were lost to follow-up.

\section{Isolation of R-CHOP-resistant DLBCL cells}

Five T25 flasks, each containing a total of $5 \times 10^{6}$ SU-DHL- 2 cells in $5 \mathrm{ml}$ of complete medium $\left(1 \times 10^{6}\right.$ cells $/ \mathrm{ml}$ ), were treated with increasing concentrations of on-and-off cycles of R-CHOP (cyclophosphamide, doxorubicin, vincristine and prednisone, combined with rituximab) exposure. The highest concentrations of cyclophosphamide, doxorubicin, vincristine and prednisone, rituximab and total R-CHOP were set at 4232, 292, 8, 588 and $5120 \mathrm{ng} / \mathrm{ml}$; the lowest concentrations were set at 264.5, 18.25, 0.5, 36.75 and $320 \mathrm{ng} / \mathrm{ml}$. Cyclophosphamide (C0768) and prednisone (P6254) were purchased from Sigma-Aldrich Corporation (MO, USA). Doxorubicin (KGA8184) and vincristine (KGA8252) were purchased from KeyGen Biotech (Nanjing, China). Rituximab was purchased from Roche Pharma (Schweiz) Ltd (Basel, Switzerland). Cyclophosphamide, doxorubicin, vincristine and rituximab were dissolved in Millipore-purified water, and prednisone was dissolved in dimethyl sulfoxide. All drugs were stored at $-80^{\circ} \mathrm{C}$.

\section{Cell-line chemosensitivity assay}

In vitro chemosensitivity was assessed using the CellTiter $96^{\circledR}$ AQueous One Solution Cell Proliferation Assay (MTS, Promega, WI, USA, G3580). Assays were performed by preincubating wells, each containing $4.44 \times 10^{4}$ tumor cells, with $20 \mu \mathrm{l}$ of MTS reagent per well for $4 \mathrm{~h}$. The plates were read at $490 \mathrm{~nm}$ for MTS, and the absorbance was corrected relative to blank wells containing reagent only. The half maximal inhibitory concentration $\left(\mathrm{IC}_{50}\right)$ was calculated by probit regression.

\section{Isolation \& identification of exosomes}

After $48 \mathrm{~h}$ of culture with FBS-depleted medium (to avoid FBS-derived exogenous exosomes' interference), exosomes were isolated from SU-DHL-2 and SU-DHL-2/R cell supernatants by using an ExoQuick-TC Kit (EXOTC50A-1, System Biosciences, CA, USA) in accordance with the manufacturer's instructions. Exosomes were isolated from serum from DLBCL patients by using an ExoQuick Kit (EXOQ20A-1, System Biosciences) in accordance with the manufacturer's instructions. The exosomes were characterized by Western blot analysis of the exosome-specific markers - TSG101 (Abcam, Cambridge, UK, ab133586, at a working dilution of 1:1000) and HSP70 (Abcam, Cambridge, UK, ab2787, at a working dilution of 1:1000). Exosomes were also identified by dynamic light scattering (DLS; Malvern Instruments, Ltd, Worcestershire, UK) and transmission electron microscopy (TEM; FEI, OR, USA). 


\section{Western blotting}

Equal amounts of proteins $(30 \mu \mathrm{g})$ were separated via 10\% SDS-PAGE and transferred to polyvinylidene difluoride membranes. After blocking, the membranes were incubated with the primary antibody at $4^{\circ} \mathrm{C}$ overnight. A horseradish peroxidase (Sigma-Aldrich Corporation) conjugated secondary antibody was used and visualized with enhanced chemiluminescence reagents.

\section{Total RNA extraction}

Total RNA was extracted from exosomes using TRIzol reagent (Invitrogen, CA, USA) according to the manufacturer's instructions. RNA samples were quantified by using a NanoDrop spectrophotometer (Thermo Scientific, MA, USA). The quality of RNA samples was assessed on a Bioanalyzer (Agilent Genomics, CA, USA).

\section{RNA sequencing}

Total RNA from each sample was used to prepare the miRNA sequencing library, which included the following steps: $3^{\prime}$-adaptor ligation; 5'-adaptor ligation; cDNA synthesis; PCR amplification; and size selection of approximately 135-155 bp PCR amplified fragments (corresponding to $\sim 15-35 \mathrm{nt}$ small RNAs). The libraries were denatured as single-stranded DNA molecules, captured on Illumina flow cells, amplified in situ as clusters and finally sequenced for 50 cycles on an Illumina NextSeq 500 (Illumina, CA, USA) system according to the manufacturer's instructions.

\section{miRNA-seq data analysis}

After sequencing, filtered raw reads were harvested as Clean Reads by using Solexa CHASTITY QC. The adaptor sequences were trimmed and the adaptor-trimmed-reads $(\geq 15 \mathrm{nt})$ were left. miRDeep2 software was used to predict the novel miRNAs from these trimmed reads. Then, the trimmed reads were aligned and merged with premiRNA databases (known premiRNAs from miRBase v21 plus the newly predicted premiRNAs) using NovoAlign software (v2.07.11). We used the most abundant isomiR, the mature miRNA annotated in miRBase and all isoforms of the miRNA ( $5 \mathrm{p}$ or $3 \mathrm{p}$ ) to calculate miRNA expression. When comparing the differentially expressed miRNA profiles between two groups, fold change and p-value were calculated and used to identify significant differentially expressed miRNAs. Differentially expressed miRNAs between two samples were filtered by fold change. Hierarchical clustering was performed. miRNA target prediction was performed by two database - TargetScan 7.1 (www.ta rgetscan.org/vert_71/) and mirdbV5 (http://mirdb.org/miRDB/), the overlapping results of two database were miRNA targets, Cytoscape software was used to obtained network of miRNAs and mRNAs which showed the relationship between miRNAs and targets. The DAVID (version 6.7, https://david-d.ncifcrf.gov/) webserver was used to perform enrichment analysis of top 10 differentially expressed miRNAs in Gene Ontology (GO) and Kyoto Encyclopedia of Genes and Genomes (KEGG) pathway. Gene lists were uploaded to DAVID with 'Official Gene Symbol' as identifier and submitted to analysis using the whole human genome as background, the p-value denotes the significance of GO terms enrichment in the DE genes. The p-value was adjusted by using Benjamini and Hochberg [14] method to avoid false positives due to chance, and p-value less than 0.05 was considered to be significantly enriched.

\section{Quantitative real-time PCR}

Reverse transcription of RNA was carried out with a PrimeScript ${ }^{T M}$ RT reagent kit (TaKaRa Bio, Inc., Otsu, Japan). The synthesized cDNA was subjected to RT-PCR to detect miRNAs using a SYBR Green fluorescence-based assay kit (TaKaRa Bio, Inc.). The primers used were 5'GCTTCGGCAGCACATATACTAAAAT3' and 5'CGCTTCACGAATTTGCGTGTCAT3' for U6; 5'GCCAACCCGTAGATCCGAT3' and 5'GTGCGTGTCGTGGAGTCG3' for miR-99a5p; 5'GCTCCCTGAGACCCTAAC3' and 5'GTGCGTGTCGTGGAGTCG3' for miR-125b-5p; 5'GGCAACCCTGTAGATCCGAA3' and 5'GTGCGTGTCGTGGAGTCG3' for miR-10a-5p; and 5'GGATACCCTGTAGAACCGAA $3^{\prime}$ and 5'CAGTGCGTGTCGTGGAGT3' for miR-10b-5p. Amplification was performed in an ABI Fast 7500 Real-Time PCR System (Applied Biosystems, CA, USA) using the following cycling program: $95^{\circ} \mathrm{C}$ for $10 \mathrm{~min}, 40$ cycles of $95^{\circ} \mathrm{C}$ for $10 \mathrm{~s}$ and $60^{\circ} \mathrm{C}$ for $60 \mathrm{~s}$. All samples were analyzed in triplicate. The relative expression levels of targeted miRNAs and U6 were calculated based on the threshold cycle $(\mathrm{Ct})$ values as ratio $=2^{-\Delta \Delta \mathrm{Ct}}$. 


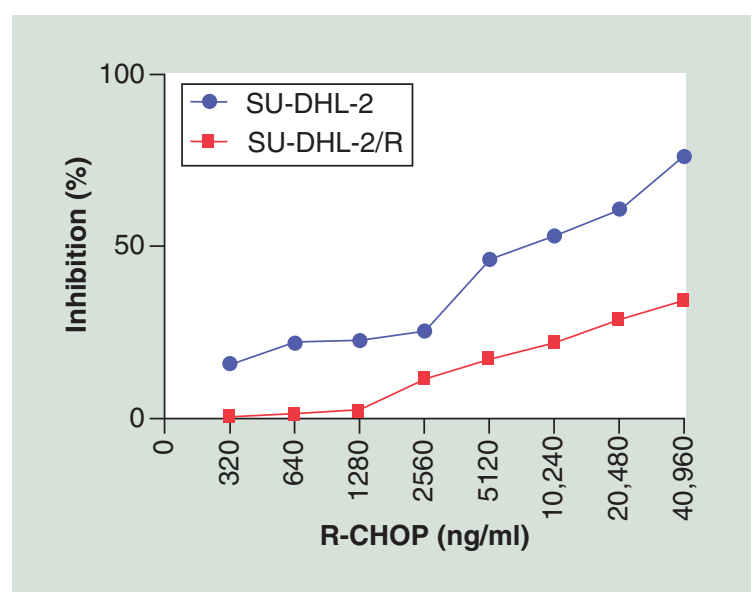

Figure 1. SU-DHL-2/R cells resistant to R-CHOP compared with SU-DHL-2 cells $(p=0.0134)$.

Statistical analysis

We used SPSS 17.0 statistical software and GraphPad Prism 6 statistic software for data analysis. The data were presented as the mean \pm standard error of the mean. miRNAs in exosomes derived from SU-DHL-2 and SUDHL-2/R cells or patient serum samples were compared using a t-test. GraphPad Prism 6 software was used to obtain receiver operating characteristic (ROC) curve and calculate the area under the curve (AUC). Survival curves were calculated according to the Kaplan-Meier method. The results were considered significant when the p-values were $<0.05$.

\section{Results}

Establishment of R-CHOP-resistant DLBCL cells

To investigate the underlying mechanisms of DLBCL chemotherapy resistance, we established the multidrugresistant DLBCL cell line - SU-DHL-2/R via on-and-off cycles of R-CHOP exposure. SU-DHL-2/R cells exhibited resistance to R-CHOP (Figure $1 ; \mathrm{p}=0.0134$ ). The $\mathrm{IC}_{50}$ of SU-DHL-2/R cells was nine-times higher than that of the parental SU-DHL-2 cells.

\section{Characterization of exosomes}

To verify successful exosome isolation, exosomes were characterized by Western blotting, DLS and TEM. These exosomes showed high concentrations of the exosome specific markers - TSG101 and HSP70 (Figure 2A). DLS showed that the mean size of the exosomes was approximately $70 \mathrm{~nm}$ (Figure 2B). These exosomes showed the typical cup-shaped morphology by TEM (Figure 2C).

miRNA profiling of exosomes from DLBCL cell lines

To identify differentially expressed miRNAs in exosomes from DLBCL cell lines, miRNA NGS was performed in the SU-DHL-2 cell and SU-DHL-2/R cell groups using Illumina NextSeq 500 technology. A total of 678 miRNAs could be detected. After comparing the miRNA profiles of the two groups, there were 37 significantly upregulated miRNAs and 17 significantly downregulated miRNAs (Figure 3). The top 10 significantly upregulated miRNAs and downregulated miRNAs with fold change $\geq 1.5$ (or $\leq 0.67$ ) and $p$-value $<0.05$ are listed in Table 1.

\section{Prediction of the miRNAs' targeted genes \& networks}

We predicted the targeted genes of the top 10 differentially expressed miRNAs by using the TargetScan 7.1 and mirdbV5 databases. Among the top 10 differentially expressed, predicted miRNAs, we excluded miRNAs with the same mature miRNA but different premiRNAs compared with other known and novel miRNAs. The targeted genes and networks are shown in Figure 4.

\section{GO \& KEGG pathway analysis}

We selected the targeted genes of the top 10 upregulated and downregulated miRNAs to perform GO and KEGG pathway analysis. The top 10 most enriched categories in Biological Process, Cellular Component and Molecular Function are shown in Figure 5. The top 10 important pathways are shown in Figure 6. 


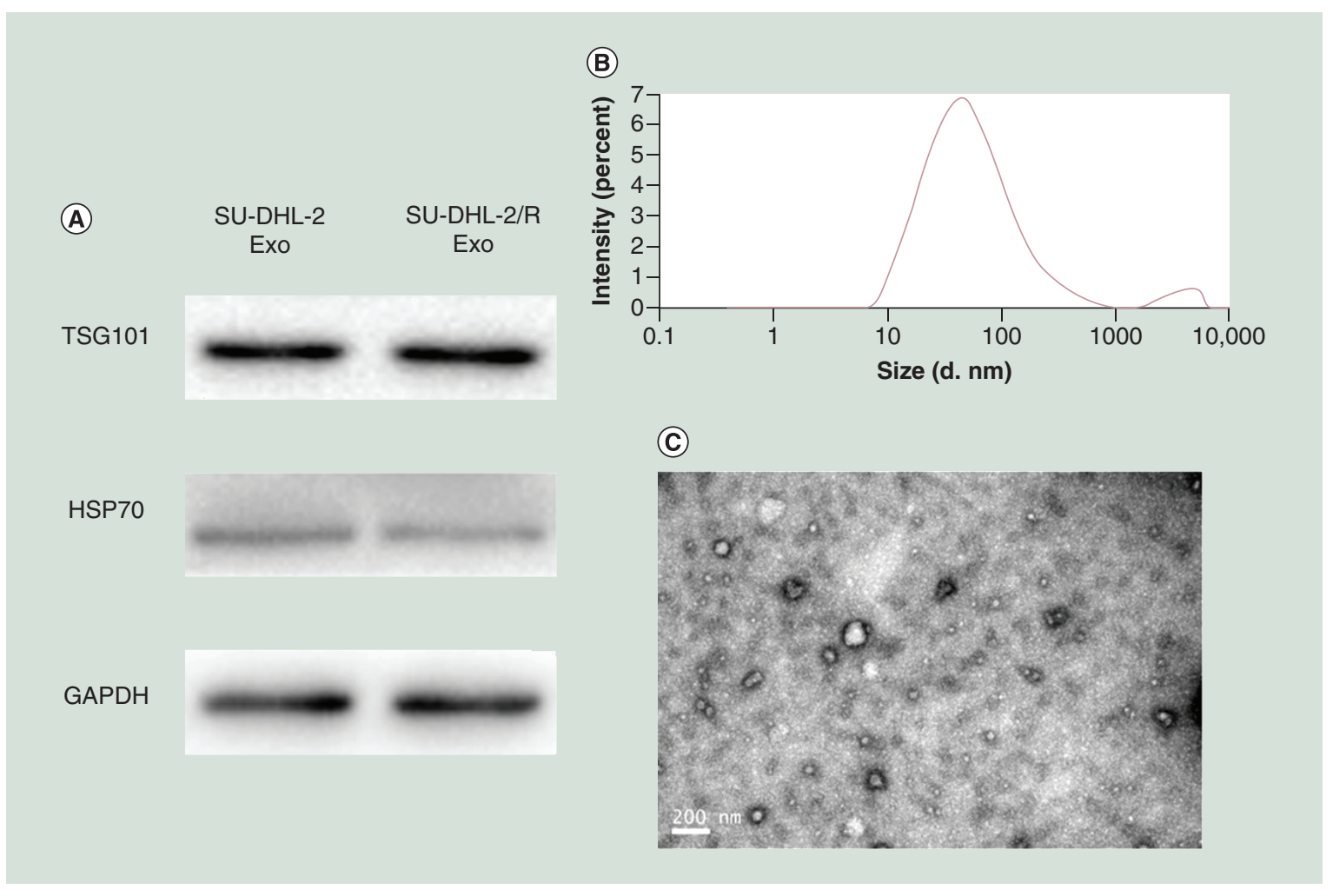

Figure 2. Characterizations of exosomes. (A) Western blotting indicating exosomes presenting the exosome specific markers - TSG101 and HSP70. (B) Dynamic light scattering revealing that the mean size of exosomes was approximately $70 \mathrm{~nm}$. (C) TEM image of exosomes showing the typical cup-shaped morphology.

Table 1. Top 10 upregulated and downregulated miRNAs.

\begin{tabular}{|llllll|}
\hline MATURE-ID & Mean (SU-DHL-2 $/$ R) & Mean (SU-DHL-2) & Fold change & p-value & Description \\
\hline hsa-miR-99a-5p & 4673 & 445.666667 & 10.485415 & 0.002221553 & Up \\
\hline hsa-miR-125b-2-3p & 82.666667 & 13.333333 & 6.2 & 0.018109233 & Up \\
\hline hsa-miR-10b-5p & 1359 & 270.666667 & 5.020936 & 0.000937769 & Up \\
\hline hsa-miR-125b-5p & 459 & 102.666667 & 4.470779 & 0.02087256 & Up \\
\hline hsa-miR-146b-3p & 69 & 17 & 4.058824 & 0.00855868 & Up \\
\hline hsa-miR-23a-3p & 1705.333333 & 450.666667 & 3.784024 & 0.043288241 & Up \\
\hline hsa-miR-1269a & 45.333333 & 14.666667 & 3.090909 & 0.033657598 & Up \\
\hline hsa-let-7c-5p & 302.333333 & 99 & 3.053872 & 0.001292 & Up \\
\hline hsa-miR-24-3p & 925.333333 & 304.333333 & 3.040526 & 0.034678486 & Up \\
\hline hsa-miR-novel-chr19_26407 & 923 & 303.666667 & 3.039517 & 0.034746447 & Up \\
\hline hsa-miR-novel-chr4_51772 & 15.666667 & 35.333333 & 0.443396 & 0.0248735 & Down \\
\hline hsa-miR-novel-chrX_72977 & 577.333333 & 1296 & 0.445473 & 0.0012718 & Down \\
\hline hsa-miR-122-5p & 810 & 1561.333333 & 0.518787 & 0.0354325 & Down \\
\hline hsa-miR-421 & 25.666667 & 48.666667 & 0.527397 & 0.0123551 & Down \\
\hline hsa-miR-144-5p & 253 & 475.333333 & 0.532258 & 0.0407786 & Down \\
\hline hsa-miR-novel-chr20_33917 & 19.333333 & 35.333333 & 0.54717 & 0.0471845 & Down \\
\hline hsa-miR-144-3p & 126 & 227.333333 & 0.554252 & 0.0177418 & Down \\
\hline hsa-miR-98-5p & 146.333333 & 264 & 0.554293 & 0.0260029 & Down \\
\hline hsa-miR-novel-chrX_73668 & 458.333333 & 812 & 0.56445 & 0.0158412 & Down \\
\hline hsa-miR-577 & 56 & 92.333333 & 0.606498 & 0.0381089 & Down \\
\hline
\end{tabular}




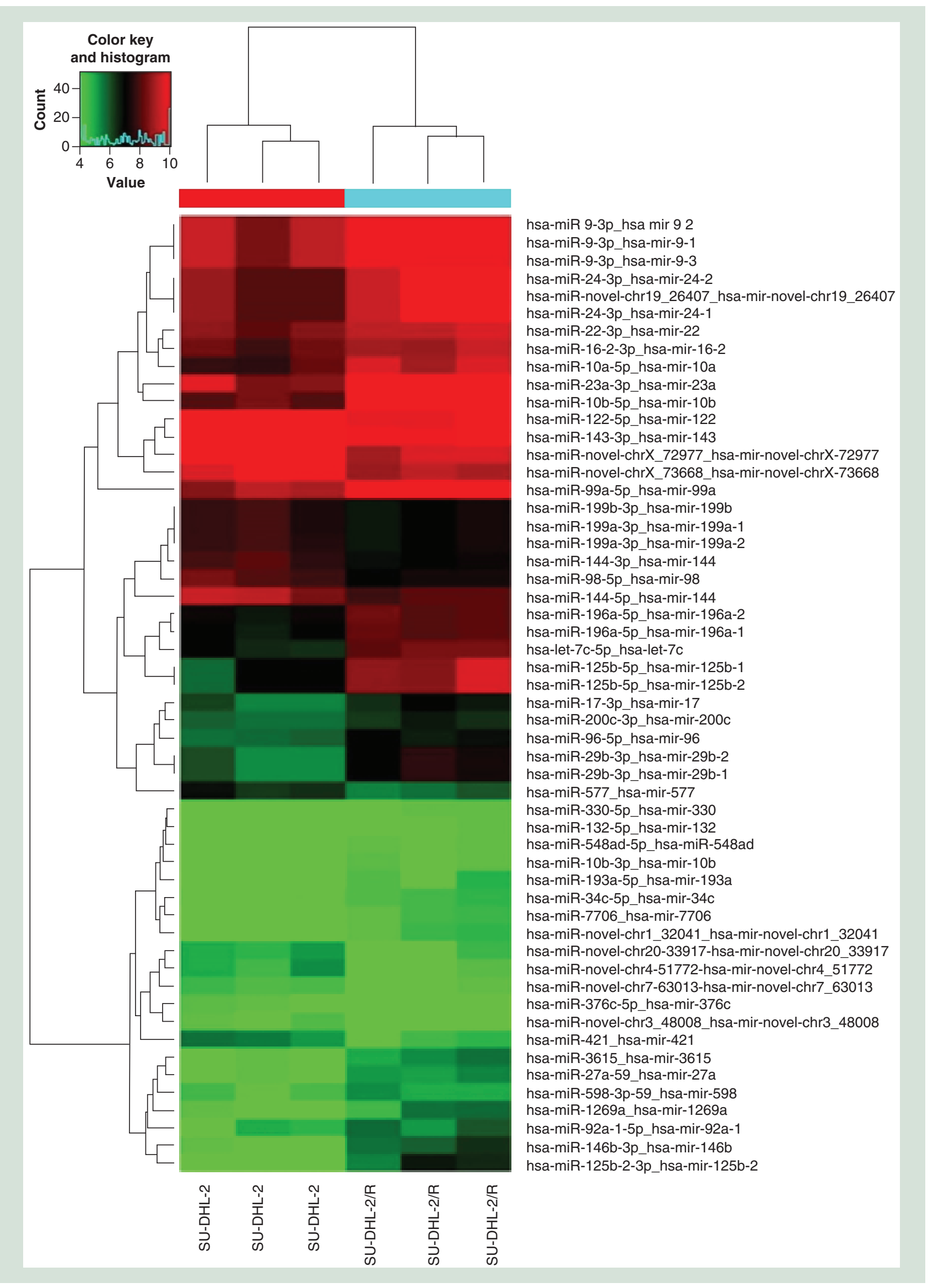

Figure 3. Heatmap showing significantly upregulated and downregulated miRNAs in the SU-DHL-2 and SU-DHL-2/R cell line groups. 


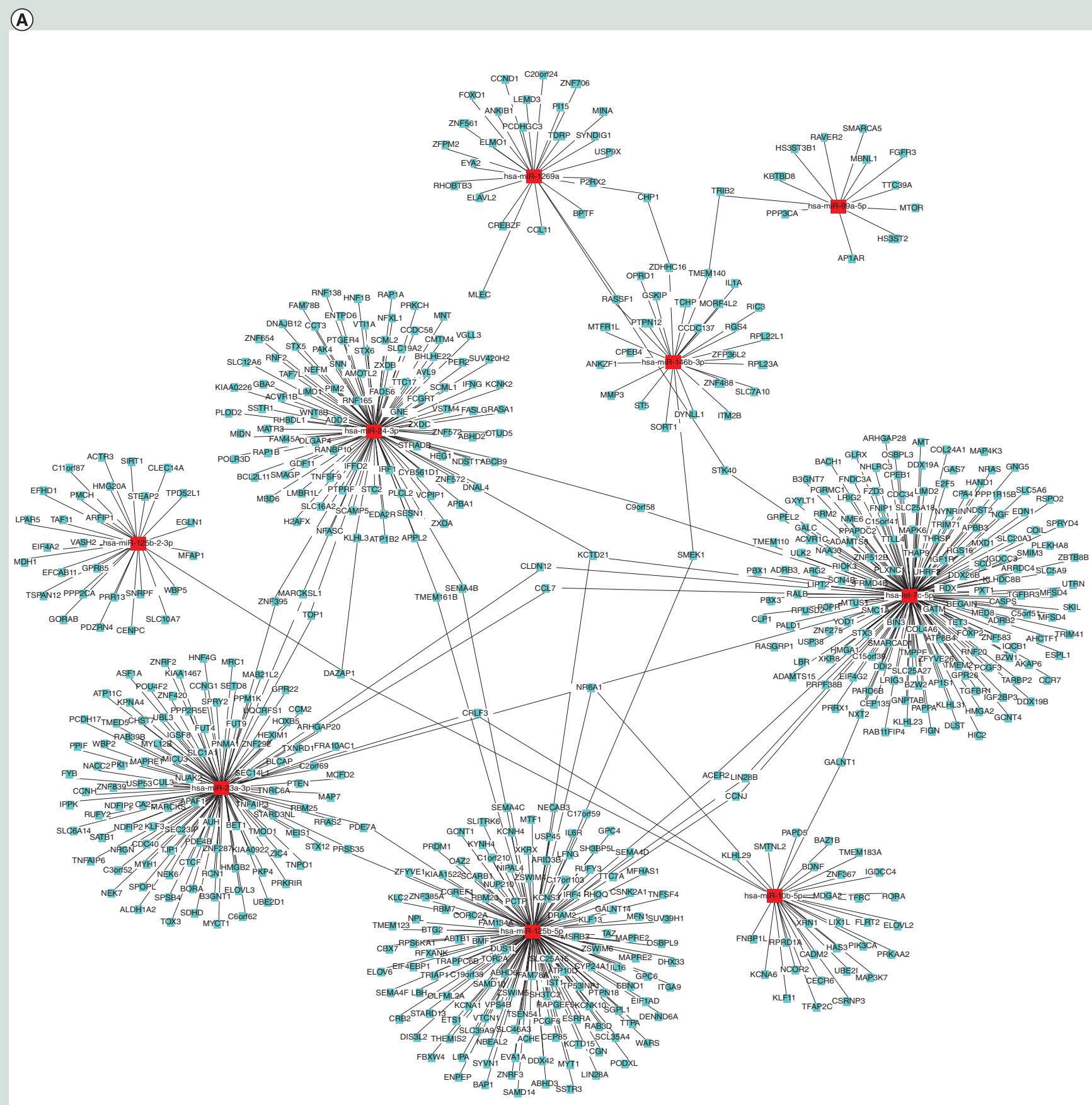

Figure 4. Prediction of the miRNAs' targeted genes and networks. (A) Prediction of upregulated miRNAs' and (B) downregulated miRNAs' targeted genes and networks.

Blue: Potential targeted gene; Red: miRNA.

Validation of miRNAs by qRT-PCR

For validation using qRT-PCR, we chose four upregulated miRNAs, and each validation was performed three-times in biological and technical replicates to avoid errors. The results confirmed significant expression of miR-99a-5p and miR-125b-5p $(\mathrm{p}<0.05)$ but not miR-10a-5p or miR-10b-5p $(\mathrm{p}>0.05)$ (Figure 7). 
(B)

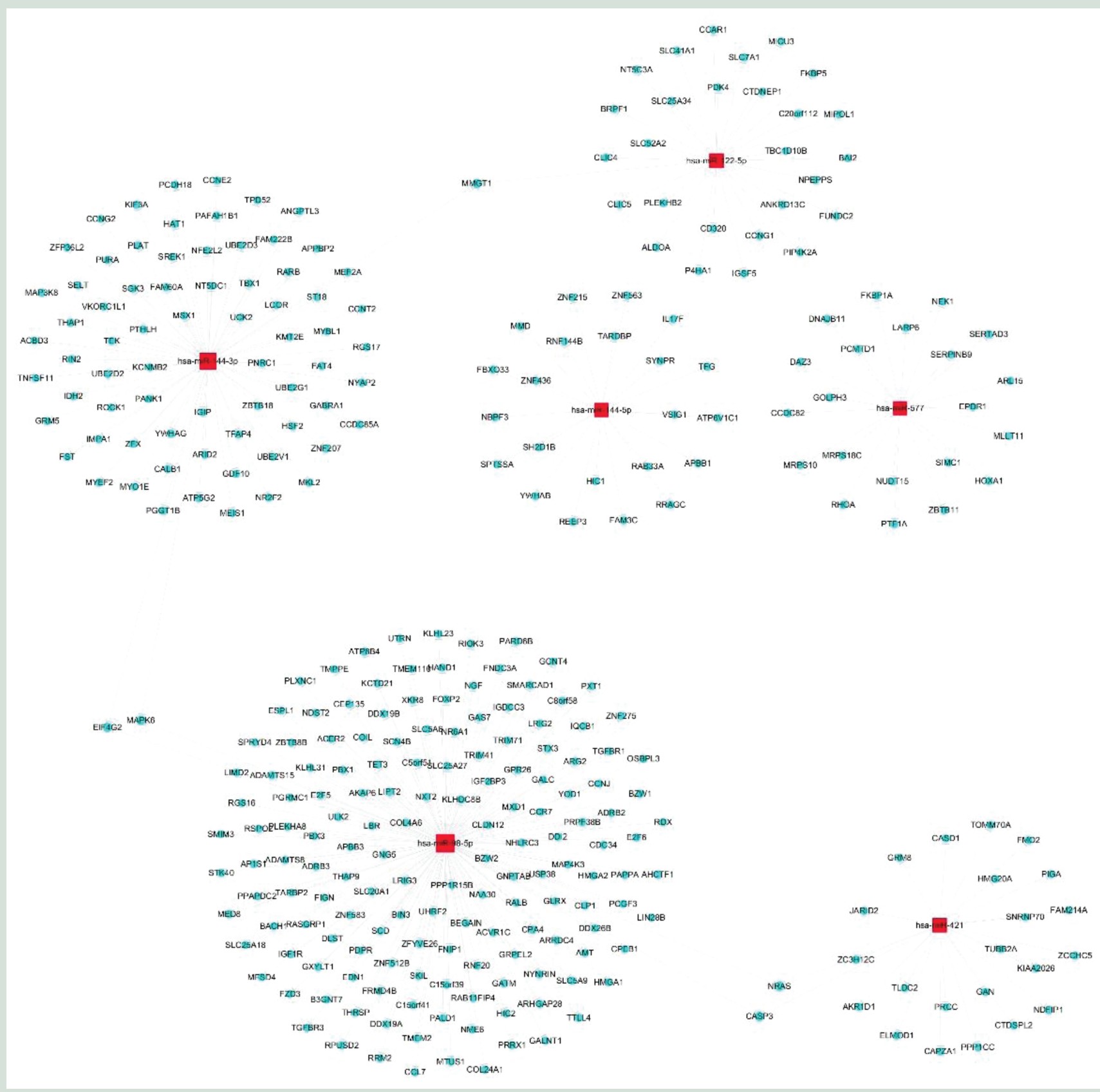

Figure 4. Prediction of the miRNAs' targeted genes and networks (cont.). (A) Prediction of upregulated miRNAs' and (B) downregulated miRNAs' targeted genes and networks.

Blue: Potential targeted gene; Red: miRNA. 


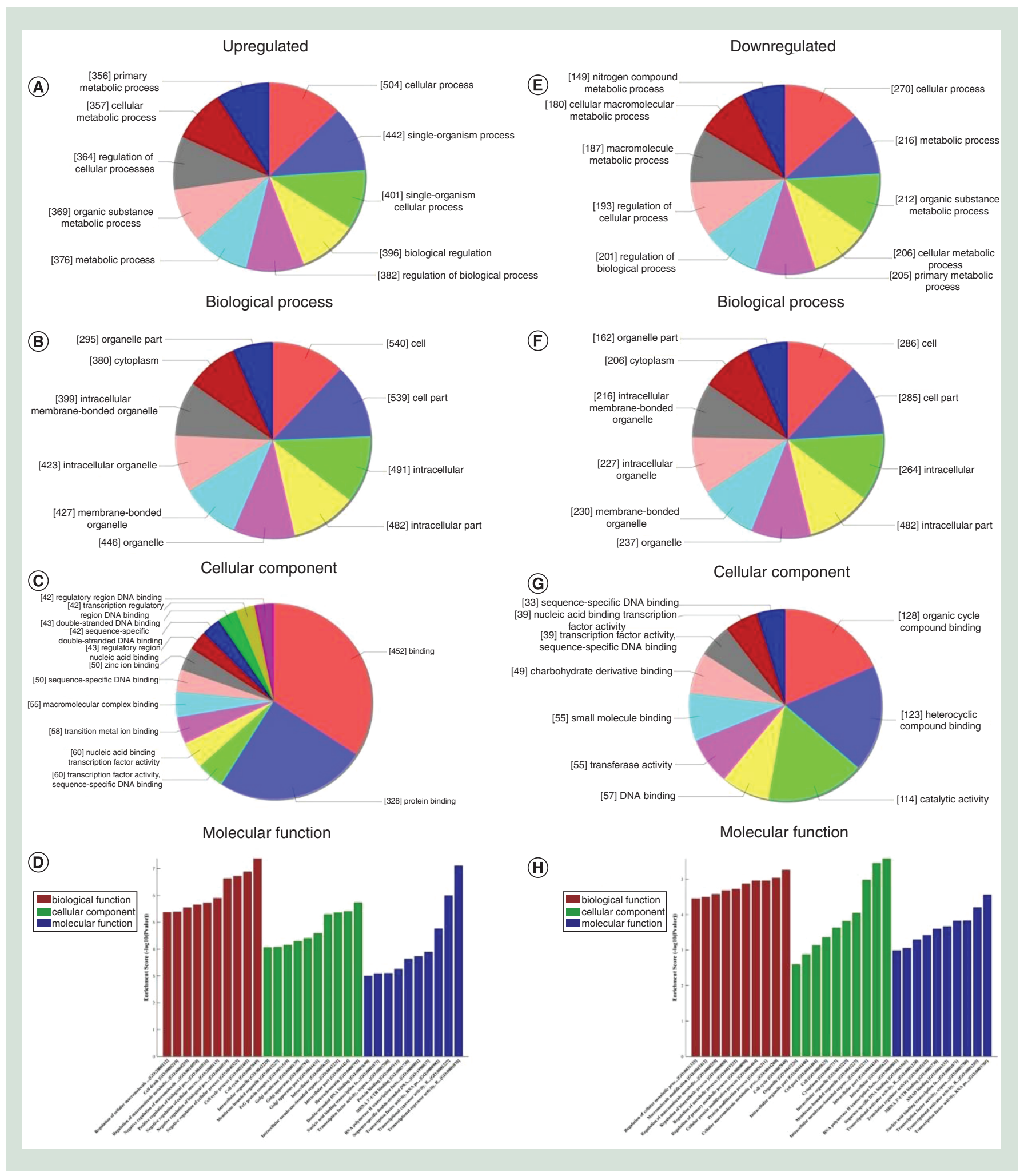

Figure 5. Gene Ontology classification of the targeted genes of the top 10 upregulated and downregulated miRNAs. The 10 most enriched categories and the enrichment scores (-log10 ( $p$-value), $p<0.05)$ in Biological Process, Cellular Component and Molecular Function are shown. (A-D) Analysis of targeted genes of upregulated miRNAs and (E-H) Analysis of targeted genes of downregulated miRNAs in Biological Process, Cellular Component and Molecular Function. 


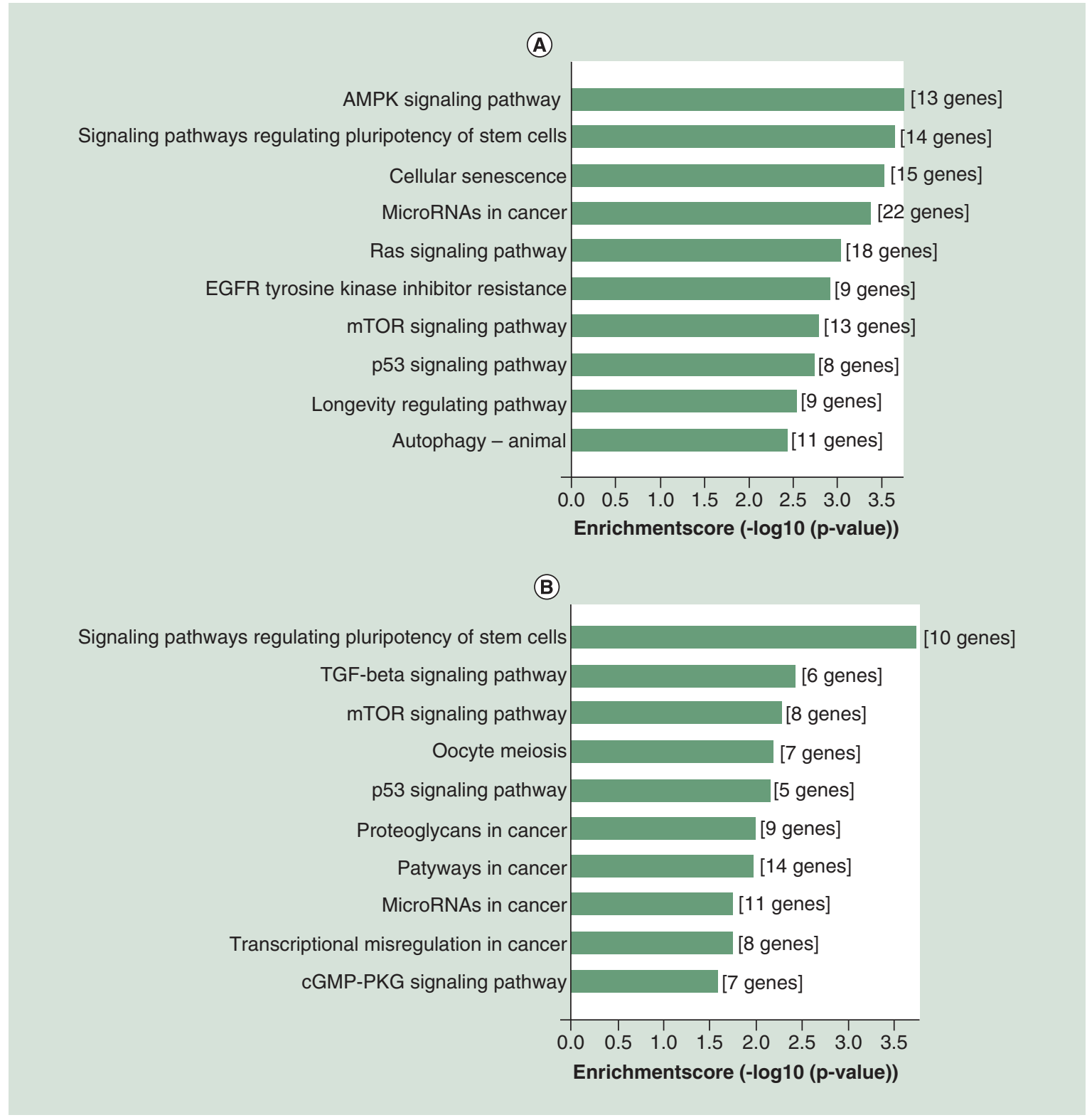

Figure 6. Kyoto Encyclopedia of Genes and Genomes analysis of the targeted genes of the top 10 upregulated and downregulated miRNAs. (A) Top 10 important pathways of the targeted genes of upregulated miRNAs and (B) the targeted genes of downregulated miRNAs.

Expression levels of exosomal miR-99a-5p/miR-125b-5p \& their correlation with clinicopathological features in DLBCL patients

We detected the expression of miR-99a-5p and miR-125b-5p in exosomes from serum samples of 116 DLBCL patients. The results showed that the expression levels of miR-99a-5p and miR-125b-5p were significantly higher in the chemoresistant group than in the chemosensitive group $(\mathrm{p}<0.001)$ (Figure 8). The expression levels of miR-99a-5p and miR-125b-5p had no relationship with patient gender, age, subtype, Ann Arbor stage, LDH, B symptoms or IPI score $(\mathrm{p}<0.05)$ (Table 2$)$.

The value of exosomal miR-99a-5p/miR-125b-5p in predicting treatment efficacy \& prognosis for DLBCL patients

We analyzed the predictive ability of exosomal miR-99a-5p/miR-125b-5p and IPI scores in distinguishing chemosensitive and chemoresistant DLBCL patients. ROC curve analysis results showed that the AUC values 


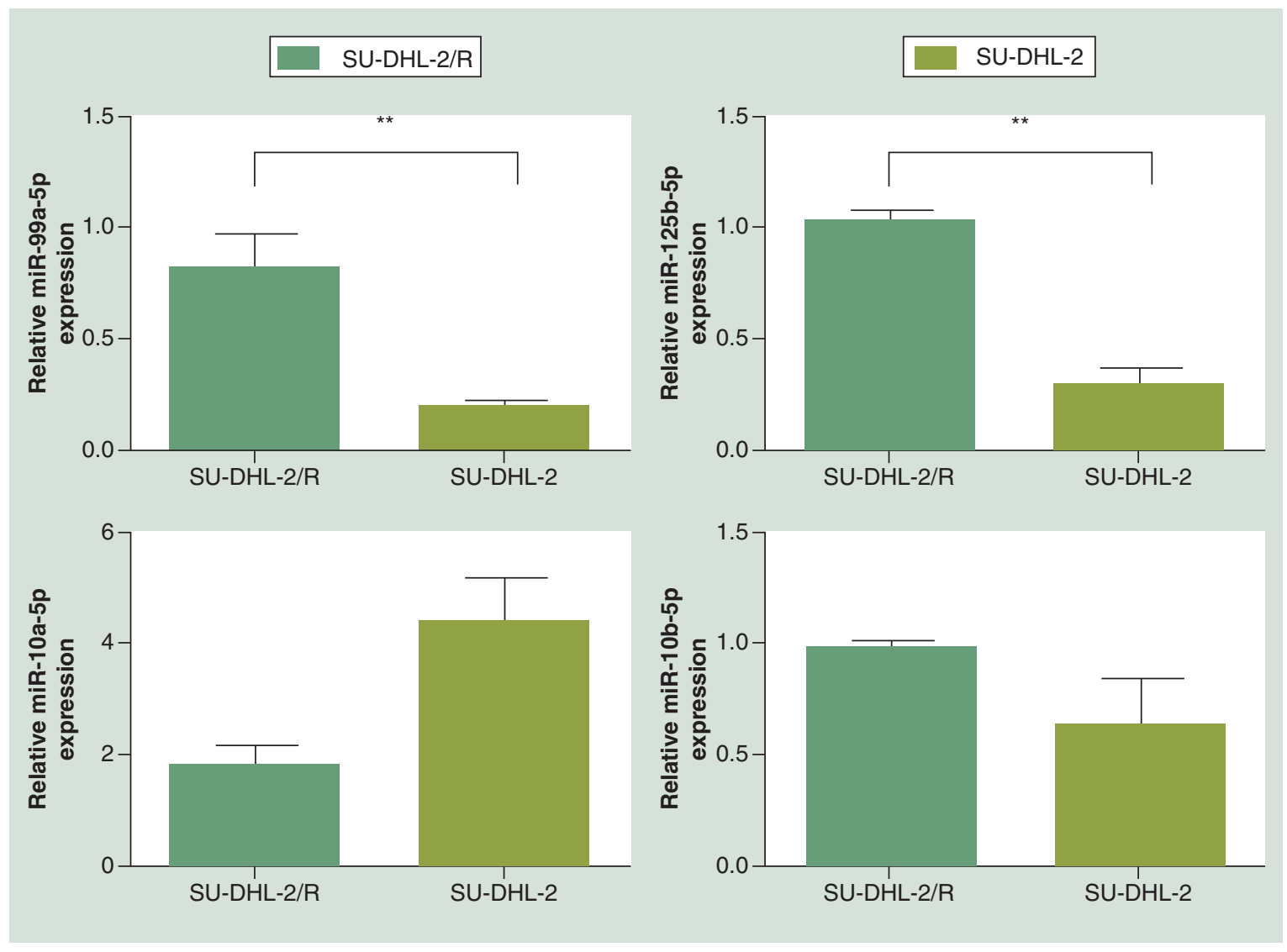

Figure 7. Quantitative real-time PCR validation of miRNAs in exosomes from SU-DHL-2/R cells and SU-DHL-2 cells. The graphs are displayed as the mean $\pm \operatorname{SEM}(n=3)$.

**p-value $<0.01$

SEM: Standard error of the mean.

(A)

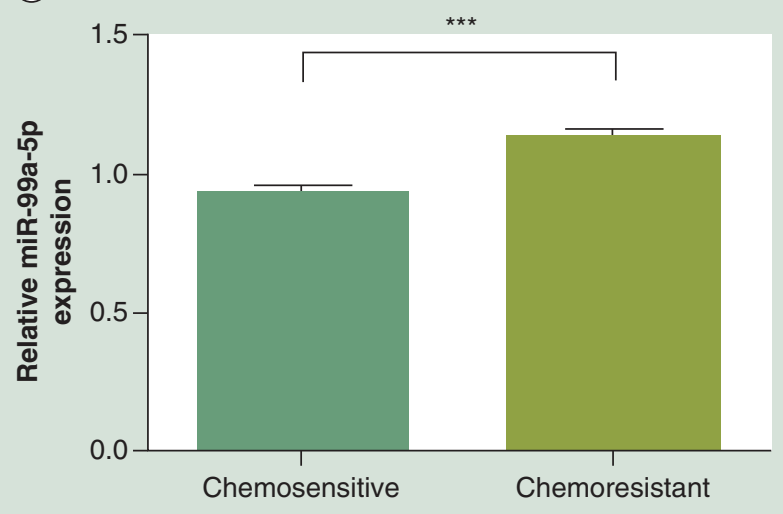

(B)

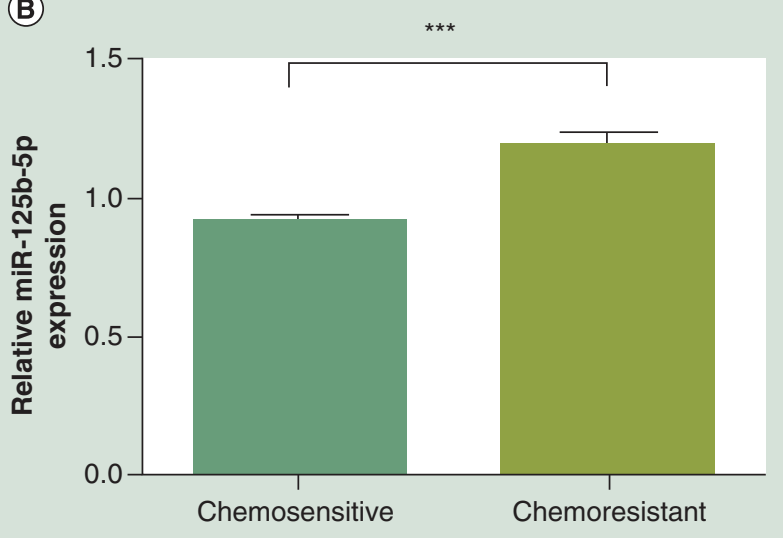

Figure 8. The expression of serum exosomal miRNAs in the chemosensitive and chemoresistant diffuse large B-cell lymphoma groups. (A) Relative expression of serum exosomal miR-99a-5p. (B) Relative expression of serum exosomal miR-125b-5p.

$* * *$ p-value $<0.001$. 


\begin{tabular}{|c|c|c|c|c|c|c|c|}
\hline \multirow[t]{2}{*}{ Characteristics } & \multicolumn{4}{|c|}{ miR-99a-5p expression } & \multicolumn{3}{|c|}{ miR-125b-5p expression } \\
\hline & Number & Low & High & $p$-value & Low & High & p-value \\
\hline \multicolumn{8}{|l|}{ Gender } \\
\hline Male & 68 & 30 & 38 & 0.397 & 31 & 37 & 0.176 \\
\hline Female & 48 & 25 & 23 & & 28 & 20 & \\
\hline \multicolumn{8}{|l|}{ Age (years) } \\
\hline$<60$ & 72 & 33 & 39 & 0.663 & 36 & 36 & 0.812 \\
\hline$\geq 60$ & 44 & 22 & 22 & & 23 & 21 & \\
\hline \multicolumn{8}{|l|}{ Subtype } \\
\hline$A B C$ & 62 & 28 & 34 & 0.603 & 30 & 32 & 0.568 \\
\hline GCB & 54 & 27 & 27 & & 29 & 25 & \\
\hline \multicolumn{8}{|l|}{ Ann Arbor Stage } \\
\hline $1+\mathrm{II}$ & 59 & 30 & 29 & 0.451 & 33 & 26 & 0.266 \\
\hline $\mathrm{III+IV}$ & 57 & 25 & 32 & & 26 & 31 & \\
\hline \multicolumn{8}{|l|}{ LDH } \\
\hline Normal & 76 & 39 & 37 & 0.246 & 42 & 34 & 0.191 \\
\hline Elevated & 40 & 16 & 24 & & 17 & 23 & \\
\hline \multicolumn{8}{|l|}{ B symptoms } \\
\hline Absent & 100 & 47 & 53 & 0.823 & 50 & 50 & 0.642 \\
\hline Present & 16 & 8 & 8 & & 9 & 7 & \\
\hline \multicolumn{8}{|l|}{ IPI score } \\
\hline Low-risk group & 68 & 36 & 32 & 0.14 & 35 & 33 & 0.295 \\
\hline Low- to medium-risk group & 25 & 9 & 16 & & 15 & 10 & \\
\hline High- to medium-risk group & 14 & 4 & 10 & & 4 & 10 & \\
\hline High-risk group & 9 & 6 & 3 & & 5 & 4 & \\
\hline
\end{tabular}

of miR-99a-5p, miR-125b-5p and IPI scores were $0.744(\mathrm{p}<0.001), 0.7802(\mathrm{p}<0.001)$ and $0.6308(\mathrm{p}<0.05)$, respectively. We calculated the combined predictive value of miR-99a-5p and IPI score, and the AUC was 0.8326 $(\mathrm{p}<0.001)$. We also calculated the combined predictive value of miR-125b-5p and IPI score, and the AUC was $0.8143(\mathrm{p}<0.001)$ (Figure 9). The combined predictive capability was better than either one of these markers alone.

The median progression-free survival (PFS) time for these 116 DLBCL patients was 19 months. Among the patients' clinicopathological features, IPI score was a significant predictor for PFS (Table 3). We used the median relative expression value for miR-99a-5p and miR-125b-5p to stratify the patients into a high miR-99a-5p group $(>1.029)$ or a low miR-99a-5p group $(<1.029)$ and a high miR-125b-5p group $(<0.993)$ or a low miR-125b-5p group $(<0.993)$. High expression levels of miR-99a-5p and miR-125b-5p contributed to unfavorable prognostic factors for DLBCL patients $(\mathrm{p}<0.001)$ (Figure 10).

\section{Discussion}

Our study presented exosome-derived miRNA profiling of DLBCL cell lines and DLBCL R-CHOP-resistant cell lines for the first time. There were 37 significantly upregulated miRNAs and 17 downregulated miRNAs detected by the NGS technique. After validation by qRT-PCR, two miRNAs (miR-99a-5p and miR-125b-5p) were observed to be significantly upregulated. Increased levels of exosomal miR-99a-5p and miR-125b-5p in serum from DLBCL patients were associated with a shorter and inferior PFS. In addition, exosomal miR-99a-5p and miR-125b-5p can be used as predictors of chemotherapeutic efficacy.

DLBCL is an aggressive non-Hodgkin lymphoma, and chemoresistance is the major obstacle for treatment. Exosomes are vesicles secreted by almost all types of cells. Exosomes play key roles in tumor chemoresistance, such as mediating drug efflux, directing tumor cells to a rescue pathway when the primary pathway is closed and reducing the penetration of anticancer drugs [15]. miRNAs are noncoding RNAs that are involved in various 
(A)

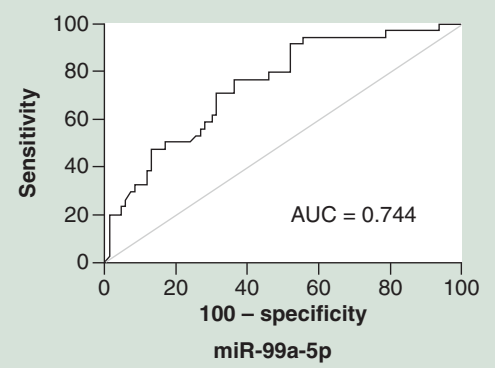

(D)
(B)

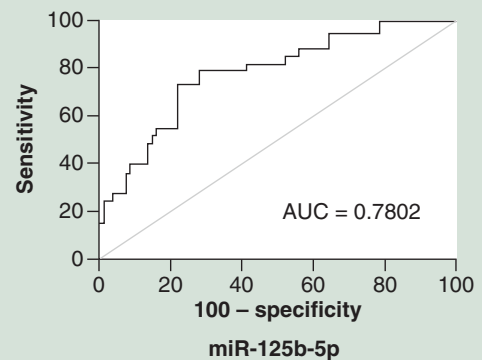

(c)

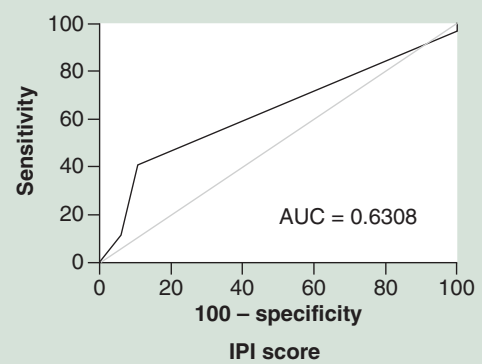

(D)

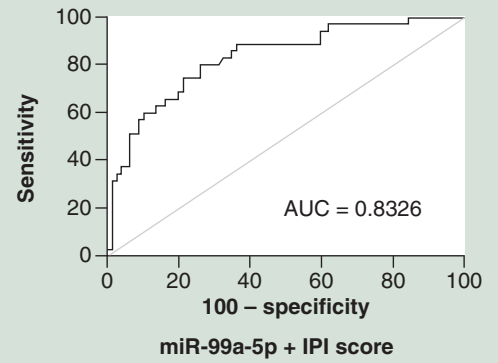

(E)

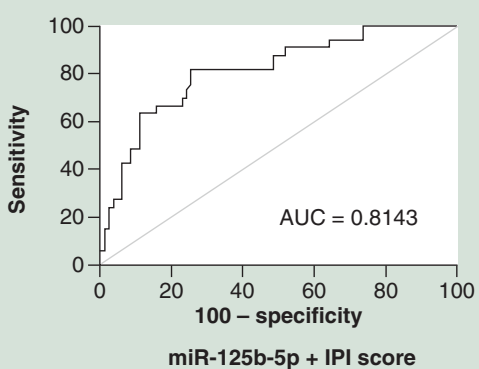

Figure 9. Receiver operating characteristic curves of biomarkers. (A) The receiver operating characteristic (ROC) curve of miR-99a-5p; the AUC was 0.744 (p-value $<0.001$ ). (B) The ROC curve of miR-125b-5p; the AUC was 0.7802 ( $p$-value $<0.001$ ). (C) The ROC curve of IPI score; the AUC was 0.6308 (p-value $=0.0268$ ). (D) The ROC curve of miR-99a-5p+IPI score; the AUC was 0.8326 ( $p$-value $<0.001)$. (E) The ROC curve of miR-125b-5p+IPI score; the AUC was 0.8143 ( $p$-value $<0.001$ ).

AUC: Area under the curve; IPI: International Prognostic Index.

(A)

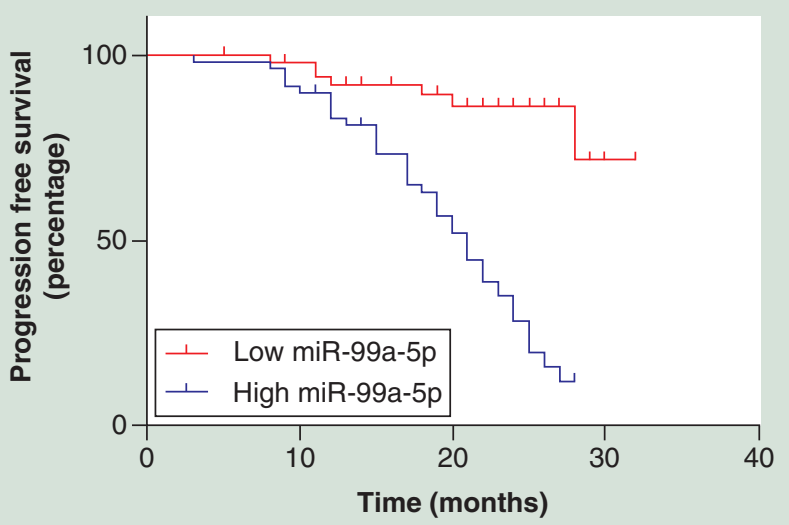

(B)

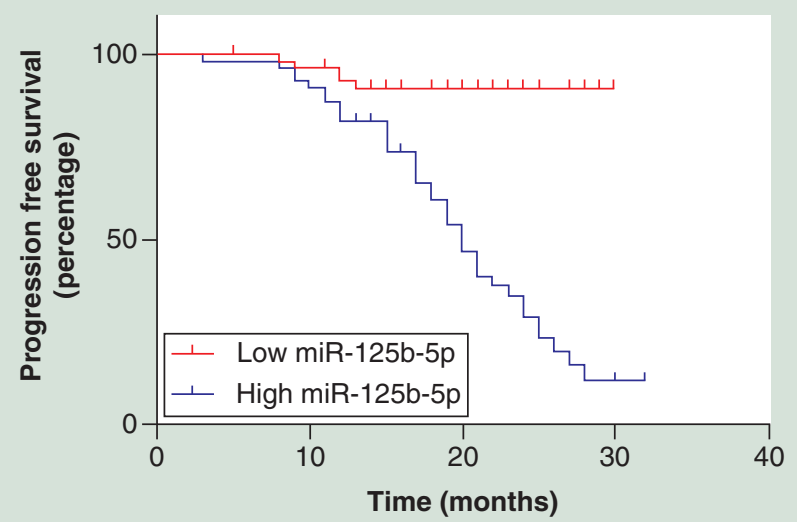

Figure 10. Association between biomarkers and progression-free survival in diffuse large B-cell lymphoma patients. (A) Kaplan-Meier curves of diffuse large B-cell lymphoma patients according to serum levels of exosomal miR-99a-5p ( $p$-value $<0.001)$. (B) Kaplan-Meier curves of diffuse large B-cell lymphoma patients according to serum levels of exosomal miR-125b-5p ( $p$-value $<0.001)$.

biological processes, and aberrant expression of miRNAs is associated with many diseases, including cancers [6]. Many miRNA signatures have been identified in cancers [16], and in DLBCL, several miRNAs, such as miR-155 and the miR-17-92 cluster, have expression patterns that distinguish DLBCL from nonmalignant B cells [17]; in addition, miR-21 expression in tumor cells [18] and serum [19] has been shown to be associated with prognosis in DLBCL patients. Exosomes contain a subset of functional miRNAs that can be delivered into cells and lead 
Table 3. Association between progression-free survival and clinicopathological features of diffuse large B-cell lymphoma

\section{patients.}

\begin{tabular}{|c|c|c|}
\hline Characteristics & PFS (months) & p-value \\
\hline Gender & & 0.672 \\
\hline Male & 25 & \\
\hline Female & 25 & \\
\hline Age (years) & & 0.266 \\
\hline$<60$ & 25 & \\
\hline$\geq 60$ & 24 & \\
\hline Subtype & & 0.713 \\
\hline $\mathrm{ABC}$ & 25 & \\
\hline GCB & Not reached & \\
\hline Ann Arbor stage & & 0.659 \\
\hline $1+11$ & 25 & \\
\hline $\mathrm{III}+\mathrm{IV}$ & 24 & \\
\hline $\mathrm{LDH}$ & & 0.064 \\
\hline Normal & 25 & \\
\hline Elevated & 21 & \\
\hline B symptoms & & 0.968 \\
\hline Absent & 25 & \\
\hline Present & Not reached & \\
\hline IPI score & & 0.005 \\
\hline Low-risk group & 27 & \\
\hline Low- to medium-risk group & 24 & \\
\hline High- to medium-risk group & 22 & \\
\hline High-risk group & 15 & \\
\hline
\end{tabular}

to tumor chemoresistance [20]. Exosomal miRNAs circulate in body fluids in a highly stable form, allowing their use as biomarkers to predict both the clinical outcome of chemotherapy and the patient's risk of developing chemoresistance [20].

In the present study, we found 678 exosomal miRNAs, including 37 upregulated and 17 downregulated miRNAs. We predicted the targeted genes of the top 10 differentially expressed miRNAs, and we selected targeted genes to perform GO and KEGG pathway analysis. In our KEGG pathway analysis, some well-known pathways that are closely related to cancer resistance were found, such as the AMPK signaling pathway, TGF- $\beta$ signaling pathway, mTOR signaling pathway and p53 signaling pathway. In cisplatin-resistant lung adenocarcinoma, autophagy is activated via activation of the AMPK/mTOR signaling pathway, which can enhance cisplatin resistance [21]. TGF- $\beta$ plays an important role in drug resistance against both targeted and conventional agents; to increase chemosensitivity, drugs that suppress TGF- $\beta$ signaling are used to treat tumors [22]. $\mathrm{p} 53$ often acts as a tumor suppressor; once it mutates, it becomes a tumor promoter and leads to chemoresistance, which can be seen in colon cancer [23]. Our KEGG pathway analysis results revealed pathways related to chemoresistance, and we can focus on these pathways in further studies of DLBCL chemoresistance.

Among the differentially expressed miRNAs, we selected four upregulated miRNAs (miR-99a-5p, miR-10b-5p, miR-125b-5p and miR-10a-5p) for validation. The validation results showed that the expression levels of miR-99a$5 p$ and miR-125b-5p were consistent with the sequencing results. In a cohort study of DLBCL patients, we found that the expression levels of both exosomal miR-99a-5p and miR-125b-5p were higher in R-CHOP chemoresistant patients than chemosensitive patients, and these miRNAs predicted prognosis and chemotherapeutic efficacy. MiR99a plays a key role in the progression of various malignant tumors, and acts as an important prognostic factor. It has been reported that the expression of miR-99a is higher in cisplatin-resistant gastric cells than cisplatin-sensitive cells, and the sensitivity of gastric cells to cisplatin was driven by miR-99a [24]. In contrast, some studies viewed miR99a-5p as a tumor suppressor that can inhibit tumor proliferation, migration and metastasis [25]. This discrepancy 
may be attributed to different sources of miRNAs, different regions of origin and different samples; more studies are needed to clarify this issue.

MiR-125b plays a key role in tumor cell proliferation, differentiation and apoptosis [26]. It was reported in previous study that the expression of miR-125b was higher in R-CHOP-resistant DLBCL patients, and miR-125b acted as an indicator for inferior prognosis [27]. In another study, miR-125b was found to be upregulated in doxorubicinresistant Ewing sarcoma, and the knock-down of miR-125b could enhance sensitivity to doxorubicin [28]. These results were consistent with the results of our study, indicating that miR-125b is an indicator for chemoresistance, and acts as an inferior prognosis factor for DLBCL patients.

\section{Conclusion}

In summary, we reported specific exosome-derived miRNA profiles of DLBCL parental cells and R-CHOP-resistant cells. Two miRNAs (miR-99a-5p and miR-125b-5p) may serve as biomarkers in DLBCL patients and can be used as predictors of prognosis and chemotherapeutic efficacy; these miRNAs may also be novel targets for treatment. However, multicenter large retrospective and/or prospective studies are needed for further investigation.

\section{Future perspective}

The roles of exosomes in cancers have been gradually realized, as noninvasive markers, exosomes can be used in diagnosis, treatment and prediction of treatment efficacy of cancers simply and accurately. Among the molecules that exosomes contain, miRNA is a research hotspot. More and more studies focus on mechanisms that exosomederived miRNAs contribute to cancer chemotherapy resistance, such as DLBCL chemotherapy resistance. In the near future, combining exosome-derived miRNAs and traditional chemotherapy agents might provide a novel strategy to overcome DLBCL chemotherapy resistance.

\section{Summary points}

\section{Background}

- Diffuse large B-cell lymphoma (DLBCL) is an aggressive non-Hodgkin lymphoma, chemoresistance is the major obstacle for its treatment.

- Dysregulation of exosome-derived miRNAs has been implied in the drug resistance of tumors. However, their roles and clinicopathological significance in DLBCL have yet to be identified.

miRNAs profiling of exosomes from DLBCL cell lines

- The next-generation sequencing technique identified a total of 54 significantly expressed (37 upregulated and 17 downregulated) miRNAs in DLBCL cell lines.

- The potential target genes of these differentially expressed miRNAs were involved in various pathways that are closely related to cancer resistance, such as the AMPK signaling pathway, TGF- $\beta$ signaling pathway, mTOR signaling pathway and $\mathrm{p} 53$ signaling pathway.

Exosomal miR-99a-5p \& miR-125b-5p as biomarkers in DLBCL

- In a DLBCL cohort, we found that the expression levels of miR-99a-5p and miR-125b-5p were significantly higher in the chemoresistant group than in the chemosensitive group.

- Exosomal miR-99a-5p and miR-125b-5p had high accuracy in predicting DLBCL patients' chemoresistance and combining with International Prognostic Index score can have a higher accuracy.

- High expression levels of miR-99a-5p and miR-125b-5p contributed to unfavorable prognostic factors for DLBCL patients.

\section{Conclusion}

- Exosome-derived miRNAs may participate in chemoresistant process of DLBCL by regulating the expression of their target genes.

- Exosomal miR-99a-5p and miR-125b-5p can serve as therapeutic and predictive biomarkers for DLBCL.

\section{Acknowledgements}

The authors thank the Department of Oncology, Xiangya Hospital of Central South University for their technical support.

\section{Authors' contributions}

Yiping Liu designed the research study. Yuhua Feng performed the research and drafted the manuscript. Meizuo Zhong and Shan Zeng participated in the literature search. Leyuan Wang analyzed the data. Ping Liu and Xiangyu Xiao collected the clinical data. All authors read and approved the final version of the manuscript. 
Financial \& competing interests disclosure

This work was supported by a grant from the National Natural Science Foundation of China (Number 81570200). The authors have no other relevant affiliations or financial involvement with any organization or entity with a financial interest in or financial conflict with the subject matter or materials discussed in the manuscript apart from those disclosed.

No writing assistance was utilized in the production of this manuscript.

Ethical conduct of research

All serum samples were obtained with the written consent of patients. The study was approved by the ethical committee of Xiangya Hospital of Central South University (number 201603172).

Open access

This work is licensed under the Attribution-NonCommercial-NoDerivatives 4.0 Unported License. To view a copy of this license, visit http://creativecommons.org/licenses/by-nc-nd/4.0/

\section{Reference}

1. Martelli M, Ferreri AJ, Agostinelli C, Di Rocco A, Pfreundschuh M, Pileri SA. Diffuse large B-cell lymphoma. Crit. Rev. Oncol. Hematol. 87(2), 146-171 (2013).

2. Swerdlow SH, Campo E, Harris NL et al. WHO Classification of Tumours of Haematopoietic and Lymphoid Tissues (4th Edition). International Agency for Research on Cancer. Lyon, France (2008).

3. Schmitz R, Wright GW, Huang DW et al. Genetics and pathogenesis of diffuse large B-cell lymphoma. N. Engl. J. Med. 378(15), 1396-1407 (2018).

4. Roschewski M, Staudt LM, Wilson WH. Diffuse large B-cell lymphoma-treatment approaches in the molecular era. Nat. Rev. Clin. Oncol. 11(1), 12-23 (2014).

5. Jiang J, Liu Y, Tang Y et al. ALDH1A1 induces resistance to CHOP in diffuse large B-cell lymphoma through activation of the JAK2/STAT3 pathway. Onco. Targets Ther. 9, 5349-5360 (2016).

6. Wang WT, Chen YQ. Circulating miRNAs in cancer: from detection to therapy. J. Hematol. Oncol. 7, 86 (2014).

7. Chitwood DH, Timmermans MC. Small RNAs are on the move. Nature 467(7314), 415-419 (2010).

8. Fan S, Chen WX, Lv XB et al. miR-483-5p determines mitochondrial fission and cisplatin sensitivity in tongue squamous cell carcinoma by targeting FIS1. Cancer Lett. 362(2), 183-191 (2015).

9. Sarkar FH, Li Y, Wang Z, Kong D, Ali S. Implication of microRNAs in drug resistance for designing novel cancer therapy. Drug Res. Updat. 13(3), 57-66 (2010).

10. Zhang X, Yuan X, Shi H, Wu L, Qian H, Xu W. Exosomes in cancer: small particle, big player. J. Hematol. Oncol. 8, 83 (2015).

11. Isola AL, Chen S. Exosomes: the messengers of health and disease. Curr. Neuropharmacol. 15(1), 157-165 (2017).

12. Valadi H, Ekstrom K, Bossios A, Sjostrand M, Lee JJ, Lotvall JO. Exosome-mediated transfer of mRNAs and microRNAs is a novel mechanism of genetic exchange between cells. Nat. Cell Biol. 9(6), 654-659 (2007).

13. Koch R, Aung T, Vogel D et al. Nuclear trapping through inhibition of exosomal export by indomethacin increases cytostatic efficacy of doxorubicin and pixantrone. Clin. Cancer Res. 22(2), 395-404 (2016).

14. Benjamini Y, Hochberg Y. Controlling the false discovery rate: a practical and powerful approach to multiple testing. J. R. Stat. Soc. B. 57, 289-300 (1995).

15. Zhao L, Liu W, Xiao J, Cao B. The role of exosomes and "exosomal shuttle microRNA" in tumorigenesis and drug resistance. Cancer Lett. 356(2 Pt B), 339-346 (2015).

16. Calin GA, Croce CM. MicroRNA signatures in human cancers. Nat. Rev. Cancer 6(11), 857-866 (2006).

17. Roehle A, Hoefig KP, Repsilber D et al. MicroRNA signatures characterize diffuse large B-cell lymphomas and follicular lymphomas. $B r$. J. Haematol. 142(5), 732-744 (2008).

18. Lawrie CH, Soneji S, Marafioti T et al. MicroRNA expression distinguishes between germinal center B cell-like and activated B cell-like subtypes of diffuse large B cell lymphoma. Int. J. Cancer 121(5), 1156-1161 (2007).

19. Lawrie $\mathrm{CH}, \mathrm{Gal} S$, Dunlop HM et al. Detection of elevated levels of tumour-associated microRNAs in serum of patients with diffuse large B-cell lymphoma. Br. J. Haematol. 141(5), 672-675 (2008).

20. Bach DH, Hong JY, Park HJ, Lee SK. The role of exosomes and miRNAs in drug-resistance of cancer cells. Int. J. Cancer 141(2), 220-230 (2017).

21. Wu T, Wang MC, Jing $\mathrm{L}$ et al. Autophagy facilitates lung adenocarcinoma resistance to cisplatin treatment by activation of AMPK/mTOR signaling pathway. Drug Des. Devel. Ther. 9, 6421-6431 (2015). 
22. Brunen D, Willems SM, Kellner U, Midgley R, Simon I, Bernards R. TGF- $\beta$ : an emerging player in drug resistance. Cell Cycle 12(18), 2960-2968 (2013).

23. Li XL, Zhou J, Chen ZR, Chng WJ. P53 mutations in colorectal cancer - molecular pathogenesis and pharmacological reactivation. World J. Gastroenterol. 21(1), 84-93 (2015).

24. Zhang Y, Xu W, Ni P, Li A, Zhou J, Xu S. MiR-99a and MiR-491 regulate cisplatin resistance in human gastric cancer cells by targeting CAPNS1. Int. J. Biol. Sci. 12(12), 1437-1447 (2016).

25. Xia M, Li H, Wang JJ, Zeng HJ, Wang SH. MiR-99a suppress proliferation, migration and invasion through regulating insulin-like growth factor 1 receptor in breast cancer. Eur. Rev. Med. Pharmacol. Sci. 20(9), 1755-1763 (2016).

26. Banzhaf-Strathmann J, Edbauer D. Good guy or bad guy: the opposing roles of microRNA 125b in cancer. Cell Commun. Signaling 12, 30 (2014).

27. Yuan WX, Gui YX, Na WN, Chao J, Yang X. Circulating microRNA-125b and microRNA-130a expression profiles predict chemoresistance to R-CHOP in diffuse large B-cell lymphoma patients. Oncol. Lett. 11(1), 423-432 (2016).

28. Iida K, Fukushi J, Matsumoto Y et al. miR-125b develops chemoresistance in Ewing sarcoma/primitive neuroectodermal tumor. Cancer Cell Int. 13(1), 21 (2013). 
